\title{
Modelo para la determinación de la ruta más corta con funciones experimentales para arcos difusos
}

Eduardo Chandomí-Castellanos, Elías N. Escobar-Gómez, Sabino Velázquez-Trujillo, Héctor R. Hernández De León, Madaín Pérez-Patricio, Carlos Venturino De Coss Pérez

Instituto Tecnológico de Tuxtla Gutiérrez, División de Estudios de Posgrado e Investigación, Tuxtla Gutiérrez, Chiapas, México

\{eduardo.chandomi, enescobarg,

sabinovelazquez1\} @hotmail. com

\{hhernandezd, mperez, cdecoss\}@ittg.edu.mx

\begin{abstract}
Resumen. En este artículo se propone resolver el problema de la ruta más corta en zonas urbanas utilizando algoritmos de rutas más corta basada en un esquema de lógica difusa, donde los tiempos de recorrido de las calles se consideran como inciertas. Para obtener un resultado de la ruta más corta más cercano a la realidad el modelo evalúa la incertidumbre relacionada con cada una de las calles o aristas de la red con el objeto de ajustar el tiempo de recorrido, es decir, se evalúa la incertidumbre para cada calle de todas las posibles rutas, multiplicando el tiempo de recorrido de la arista por un coeficiente de ajuste. Para cada calle se consideran 3 variables difusas: Estado de las Calles, Zonas de Tráfico e Intensidad de Lluvia, y cada variable está formada con funciones de pertenencia calculadas de manera experimental. Las funciones de pertenencia se determinan por medio de entrevistas, encuestas a expertos en distribución física y datos estadísticos, incluyendo el principio de consistencia propuesto por Zadeh [20]. Para la validación del modelo se comparan los tiempos de recorrido obtenidos con el modelo propuesto con los obtenidos con funciones de pertenencia clásicas (funciones trapezoidales y triangulares) con tiempos de recorrido obtenidos en un muestreo.
\end{abstract}

Palabras clave: lógica difusa, ruta más corta, tiempo de recorrido incierto, ajuste de tiempo, funciones de pertenencia experimentales.

\section{Model for Determining the Shortest Path with Experimental Functions for Fuzzy Arcs}

\begin{abstract}
In this paper, we propose to solve the shortest route problem in urban areas using the shortest path algorithms based on a fuzzy logic scheme, where the street's travel times are uncertain. To get a result of the shortest route closer to reality, this model tests the uncertainty level for each one of the edges within the network in order to adjust the travel time that is to say the uncertainty is evaluated for each street of all possible routes, multiplying the edge's travel time by an adjustment coefficient. For each street 3 fuzzy variables are considered: State of the Streets, Traffic zones and, Rain intensity. Each variable based in one membership function estimated experimentally basis and determined through interviews, surveys of experts in physical distribution and statistical data,
\end{abstract}


including Zade's consistency principle [20]. For model validation, the travel times got with the proposed model are compared with those got with classical membership functions (trapezoidal and triangular functions) and with sampled travel times.

Keywords: fuzzy logic, shortest path, uncertain travel time, time adjustment, experimental membership functions.

\section{Introducción}

El objetivo principal de toda empresa de distribución es que los productos lleguen al lugar adecuado, en el tiempo determinado, en buenas condiciones y a un costo competitivo [12]. Los costos de un producto generalmente varían dependiendo del tipo de producto que se va a comercializar, el proceso de manufactura empleado y las condiciones del proceso de distribución. En la literatura se reporta que los costos de distribución representan en algunos casos un porcentaje mayor al $30 \%$ del valor del producto [2]. El costo de distribución cambia proporcionalmente al precio del combustible, a los costos de almacenaje de la mercancía y de mantenimiento del vehículo.

En el proceso de distribución la flotilla de repartidores debe transitar por diversas partes de la ciudad, en horarios y condiciones ambientales diferentes. En ocasiones esto provoca que los tiempos de reparto, incluso para la misma ruta, cambien considerablemente; ocasionando en muchos casos la demora en la entrega del producto, y mayores gastos de combustible y de mantenimiento [4].

Para la teoría de redes, el problema de la ruta más corta es encontrar el camino con la mínima distancia, tiempo o costo desde un origen a un destino [11]. Actualmente existen métodos que ayudan a determinar la ruta más corta [5], que facilitan la toma de decisión; [7] propone resolver el problema de la ruta más corta dado un mapa de carreteras en el que se encuentre marcado la distancia de cada par de intersecciones de un solo nodo origen a cualquier nodo destino. En [9] se propone un algoritmo más general que el de Dijkstra, debido a que determina la ruta más corta entre todos los pares de nodos o vértices del grafo, es decir de un nodo origen cualesquiera a un nodo destino cualesquiera, en [3], a diferencia de los ya mencionados, se toman en cuentan pesos negativos.

Existen otros métodos para el mismo fin, como, la programación lineal; la cual consiste básicamente en la construcción, solución y análisis del modelo lineal de un problema de ruta más corta [1].

Aplicando alguno de los algoritmos antes mencionados, para la selección de rutas, se observa que los tiempos analizados no son precisos, esto se debe a que las metodologías tradicionales consideran a los datos de manera determinista, siendo que las variables de entrada tienen un comportamiento incierto, provocando que los resultados no se apeguen a la realidad.

En la actualidad existen modelos que procesan información de manera imprecisa, difusa, con variables de entrada que tiene un comportamiento incierto [15], como el presentado por [19], que mejora el algoritmo de Floyd al representar las operaciones de la función canónica con números borrosos triangulares que se basan en el método de representación de la integración gradual media y lo utiliza para obtener un algoritmo 
de ruta más corta difusa. En [6] su investigación lo resuelve utilizando el algoritmo de Dijkstra y funciones de pertenencia triangulares y trapezoidales.

\section{Método propuesto}

Los sistemas de inferencia difusa se basan en los conceptos de la teoría difusa; en la actualidad las aplicaciones varían en áreas como el control automático [10], el diagnóstico de fallas [17], la clasificación de datos, el análisis de decisiones y la robótica [16], entre otros.

En esta investigación, con la implementación de lógica difusa se obtiene un ajuste difuso que adecua el tiempo de recorrido estimado o peso de la arista al tiempo de recorrido real. En [8] presentan un esquema general del concepto de lógica difusa; basados en este esquema se estructura el modelo propuesto, el cual está formado por los cinco elementos mostrados en la Figura 1.

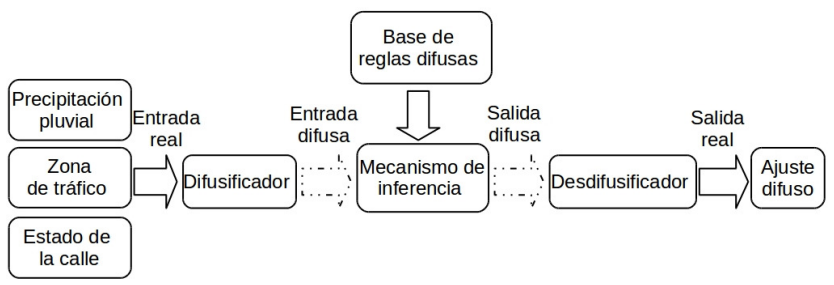

Fig. 1. Elementos de la implementación de lógica difusa.

\subsection{Variables lingüísticas}

Con base en un estudio realizado a operarios de vehículos de distribución, se detectaron 51 variables que afectan el proceso de distribución. En el análisis se determinan variables cuyo comportamiento es incierto, con mayor frecuencia y con una relación importante en afectación en el proceso de reparto. En los párrafos siguientes se describen las variables difusas y los conjuntos difusos que las forman. Los conjuntos difusos de las tres variables se determinaron de forma experimental, con un coeficiente de correlación, $R^{2}$, mayor al $95 \%$, representando el porcentaje de variación de respuesta que explica la relación de los datos reales con las funciones calculadas.

Intensidad de Lluvia (IL). Como variable lingüística, la intensidad de lluvia es la cantidad de precipitación pluvial que provoca una disminución de visibilidad y de velocidad; esta variable está medida en milímetros.

En esta variable, con base en el manual de uso de términos meteorológicos de AEMET (Agencia Estatal de Meteorología de España) [13], se consideran seis casos, los cuales definen los conjuntos difusos.

- Sin lluvia: el nivel de variable se considera conjunto difuso "sin lluvia", cuando no hay precipitación pluvial.

- Lluvia débil, el nivel de variable se considera conjunto difuso "lluvia débil", cuando representa una precipitación de 0.1 a $2 \mathrm{ml}$. 
- Lluvia normal: el nivel de variable se considera conjunto difuso "lluvia normal" cuando se tiene una precipitación pluvial de 2.1 a $15 \mathrm{ml}$

- Lluvia fuerte: el nivel de variable se considera conjunto difuso "lluvia fuerte" cuando se presenta una precipitación de 15.1 a $30 \mathrm{ml}$.

- Lluvia muy fuerte: el nivel de variable se considera conjunto difuso "lluvia muy fuerte" cuando representa una precipitación de entre 30.1 a $60 \mathrm{ml}$.

- Lluvia intensa: el nivel de variable se considera conjunto difuso "lluvia intensa" cuando la precipitación pluvial es mayor a los $60 \mathrm{ml}$.

Para calcular las funciones de pertenencia de cada conjunto difuso se utilizan los datos de precipitación pluvial proporcionada por la Coordinación General del Servicio Meteorológico Nacional (CGSMN) de la Comisión Nacional del Agua (CONAGUA), registrada en el observatorio y en la estación meteorológica automática ESIME (Estación Sinóptica Meteorológica), los datos son recopilados de 1980 a 2010 en la ciudad de Tuxtla Gutiérrez, Chiapas, México; a estos datos se aplica el principio de consistencia propuesto por Zadeh [20]. En la Figura 2 se observan las funciones de pertenencia deducidas a partir de los datos históricos.

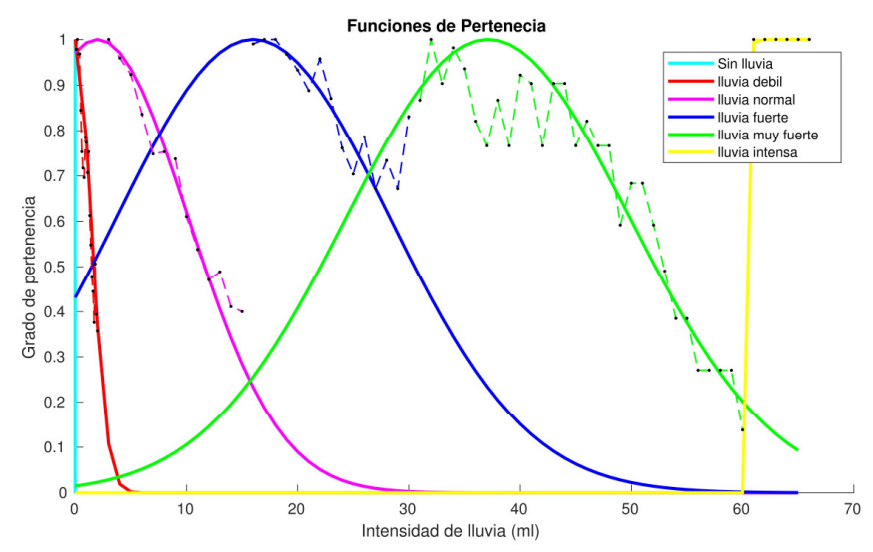

Fig. 2. Funciones de pertenencia de la variable Intensidad de Lluvia obtenidas con datos históricos.

Como se observa en la Figura 2, el primer conjunto difuso (Sin lluvia) tiene un comportamiento representado por un Singleton, ver Ecuación 1. Los siguientes cuatros conjuntos difusos presentan un comportamiento descrito mediante la distribución Gaussiana, ver Ecuación 2, 3, 4 y 5. El último conjunto difuso de la variable lluvia presenta un comportamiento similar al de la distribución Gamma, ver Ecuación 6:

$$
\mu_{\{\sin \text { lluvia }\}}(x ; a)= \begin{cases}0, & x=a, \\ 1, & x \neq a .\end{cases}
$$




$$
\begin{gathered}
\mu_{\{\text {débil }\}}(x)=\left\{\begin{array}{cc}
e^{-k\left(x^{2}\right),} & 0 \leq x \leq b, \\
0, & b>x .
\end{array}\right. \\
\mu_{\{\text {normal }\}}(x)=\left\{\begin{array}{cc}
e^{-k(x-m)^{2}}, & 0 \leq x \leq c, \\
0, & c>x .
\end{array}\right. \\
\mu_{\{\text {fuerte }\}}(x)=\left\{\begin{array}{cc}
e^{-k(x-n)^{2},} & b<x \leq d, \\
0, & 0 \leq b, d>e .
\end{array}\right. \\
\mu_{\{\text {muy fuerte }\}}(x)=\left\{\begin{array}{cc}
e^{-k(x-o)^{2}}, & c \leq x \leq e, \\
0, & 0<c .
\end{array}\right. \\
\mu_{\{\text {intensa }\}}(x)=\left\{\begin{array}{cc}
1-e^{-k(x-p)^{2},} & d \leq x \leq e, \\
0, & 0 \leq d .
\end{array}\right.
\end{gathered}
$$

Estado de las Calles (EC). La variable estado de las calles es definida por la cantidad y tamaño de obstáculos que fijan la velocidad del vehículo, es considerada difusa interpretando un nivel para cada estado de la calle:

- Estado de la calle buena: es definida por la cantidad y tamaño de obstáculos, que permiten conducir a una velocidad constante.

- Estado de la calle regular: representa la cantidad y tamaño de obstáculos que para que el conductor pueda esquivar el obstáculo debe reducir considerablemente la velocidad.

- Estado de la calle mala: es descrito como la cantidad y tamaño de obstáculos, que reducen casi por completo la velocidad pasando obligadamente sobre los obstáculos.

Se realizó un estudio para determinar el tipo de obstáculo y la cantidad que normalmente están presentes en una calle; determinándose que, en las calles de la ciudad de Tuxtla Gutiérrez, Chiapas, México comúnmente se encuentran 1, 2 o 3 topes; 1,2 o 3 baches, cada uno con el nivel de pequeño, mediano y grande; además de alcantarillado en mal estado, deformaciones de las calles provocadas por hundimientos, reparaciones o mantenimientos, cada uno con un nivel parcial, a mitad y total.

Posteriormente, se encuestaron a 200 operarios de reparto de la ciudad de Tuxtla Gutiérrez, Chiapas, México para determinar el grado de pertenencia de cada uno de los tres conjuntos difusos que se muestra en la Figura 3.

En la Figura 3 se observa que el eje de las abscisas representa el universo en discurso, el cual es determinado en el estudio de obstáculos, alcantarillas y calles realizado; y el eje de las ordenadas representa el grado de pertenencia en el intervalo $[0,1]$ de las funciones de membresía. 


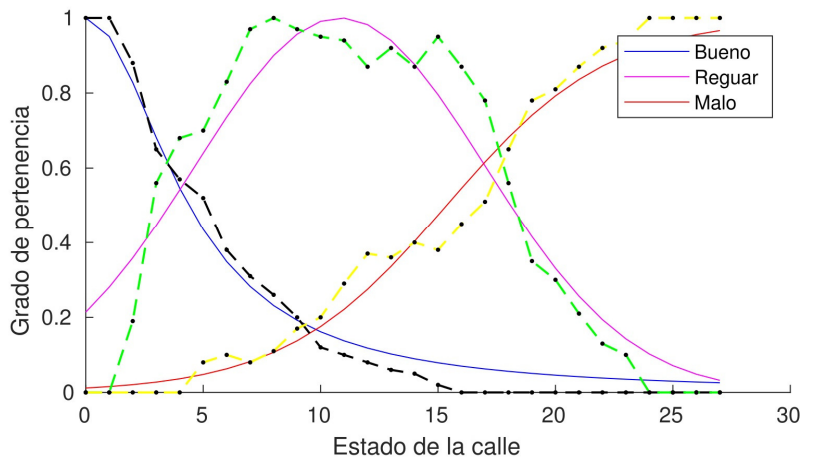

Fig. 3. Funciones de pertenencia de la variable Estado de la Calle obtenidas con datos muestreados.

Para determinar el grado de pertenencia se aplica el método horizontal, el que se basa en las respuestas de un grupo de N "expertos", ver Ecuación 7. El entrevistado responde la pregunta, “¿Puede x ser considerado compatible con el concepto A?”, Sólo se acepta un "Sí (1)" o un "No (0)".

$$
\mu_{x}=\frac{\text { Respuestas afirmativas }}{N},
$$

donde: $\mathrm{x}=$ Cada elemento $\mathrm{x} \in \mathrm{X}$ con su grado de pertenencia a $\mu, \mathrm{N}=\mathrm{El}$ número total de encuestados.

Con el análisis de la información recabada en las encuestas realizadas se determinaron el universo en discurso y los grados de pertenencia de cada conjunto difuso. El primer conjunto difuso, "bueno", es representado mediante la distribución Pseudo-exponencial, ver Ecuación 8; el segundo conjunto, "regular", tiene un comportamiento descrito mediante la distribución Gaussiana, ver Ecuación 9; el último conjunto, "malo", se describe mediante la distribución Sigmoidal, ver Ecuación 10.

$$
\begin{aligned}
& \mu_{\{\text {bueno }\}}(x)=\left\{\begin{array}{cc}
\frac{1}{1+k x^{2}}, & 0 \leq x \leq a, \\
0, & x>a .
\end{array}\right. \\
& \mu_{\{\text {regular }\}}(x)=\left\{e^{-k(x-m)^{2}}, \quad 0 \leq x \leq b .\right. \\
& \mu_{\{\text {malo }\}}(x)=\left\{\begin{array}{cl}
\frac{1}{1+e^{-k}(x-n)^{2}}, & a \leq x \leq b, \\
0, & x<b .
\end{array}\right.
\end{aligned}
$$

Zona de Tráfico $(\mathbf{Z T})$. Se define la variable zona de tráfico como el flujo de vehículos que transitan por una calle a cierta hora. Para esta variable se consideran tres casos y cada uno de estos define a un conjunto difuso: 
- Zona de tráfico ligero: se considera que la variable difusa zona de tráfico es "ligero" cuando el flujo de vehículos por la calle es rápido.

- Zona de tráfico medio: se considera que la variable difusa zona de tráfico es "medio" cuando el flujo de vehículos es semi-lento.

- Zona de tráfico pesado: se considera que la variable difusa zona de tráfico es "medio" cuando el flujo de vehículos es semi-lento.

Para determinar las zonas que, en la ciudad de Tuxtla Gutiérrez, Chiapas, México, presentan mayor tráfico se identificaron zonas que en cierta hora tienen mayor afluencia de vehículos, como son: escuelas, bancos, supermercados o centros comerciales y cruceros. Para determinar el grado de pertenencia de las zonas con respecto al horario se realizó una encuesta a conductores de transporte de la cuidad. Las encuestas nos proporcionan los valores del grado de pertenencia y el universo en discurso, por consiguiente, obtenemos la función de pertenencia, ver Figura 4.

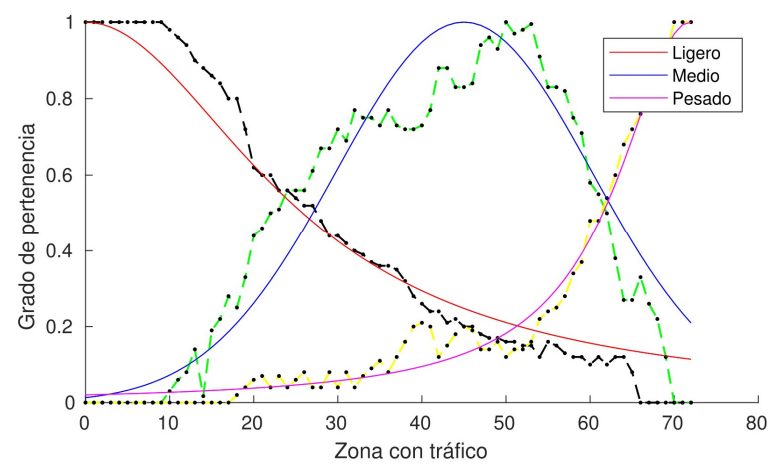

Fig. 4. Funciones de pertenencia de la variable Zona de Tráfico obtenidas con datos muestreados.

Para determinar la función de pertenencia se aplica el método horizontal, ver Ecuación 7. Para el primero y tercero conjunto difuso, "ligero" y "pesado", se determina que los datos se comportan de acuerdo a la distribución Pseudo-exponencial, ver Ecuación 11 y 13; para el segundo conjunto difuso, "medio", se establece que el comportamiento de los datos sigue una distribución Gausiana, ver Ecuación 12.

$$
\begin{gathered}
\mu_{\{\text {ligero }\}}(x)=\left\{\begin{array}{cc}
\frac{1}{1+k x^{2}}, & 0 \leq x \leq a, \\
0, & x>a .
\end{array}\right. \\
\mu_{\{\text {medio }\}}(x)=\left\{\begin{array}{cc}
e^{-k(x-m)^{2}}, & 0 \leq x \leq b .
\end{array}\right. \\
\mu_{\{\text {pesado }\}}(x)=\left\{\begin{array}{cc}
\frac{1}{1+e^{-k(x-n)^{2}}}, & a \leq x \leq b, \\
0, & x<a .
\end{array}\right.
\end{gathered}
$$




\subsection{Proceso de difusificación}

Es el proceso por el cual a las entradas reales (IL, EC y ZT) se les asigna un valor difuso (valores difusos de entrada), equivalente al grado de pertenencia; para obtener este valor difuso se utilizan las funciones correspondientes a cada conjunto difuso.

\subsection{Base de reglas difusas}

Quizás la forma más común de representar el conocimiento humano es formar expresiones de lenguaje natural del tipo; "Si" la premisa (antecedente), "Entonces" conclusión (consecuente) [18]. Estas reglas representan el conocimiento del experto en distribución física, el modelo propuesto posee tres variables difusas, dos con tres etiquetas lingüísticas y una con seis, que conforman un total de 54 reglas difusas. En la Tabla 1 se presentan las reglas que se usan para el proceso de inferencia. Ejemplo (ver regla 1): Si IL es Sin Lluvia, el EC es Bueno y ZT es Ligero entonces el AD es Muy Rápido.

Tabla 1. Base de reglas difusas.

\begin{tabular}{|c|c|c|c|c|c|c|c|c|c|c|}
\hline & \multicolumn{9}{|c|}{ Estado de las Calles } \\
\hline & & \multicolumn{3}{|c|}{ Bueno } & \multicolumn{3}{|c|}{ Regular } & \multicolumn{3}{|c|}{ Malo } \\
\hline & & \multicolumn{3}{|c|}{ Zona de Tráfico } & \multicolumn{3}{|c|}{ Zona de Tráfico } & \multicolumn{3}{|c|}{ Zona de Tráfico } \\
\hline & & Ligero & Medio & $\begin{array}{l}\text { Pesado } \\
\end{array}$ & Ligero & Medio & Pesado & Ligero & Medio & $\begin{array}{l}\text { Pesado } \\
\end{array}$ \\
\hline \multirow{6}{*}{ 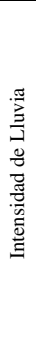 } & $\begin{array}{c}\text { Sin } \\
\text { Lluvia }\end{array}$ & $\begin{array}{c}\text { Muy } \\
\text { Rápido }\end{array}$ & Rápido & Lento & Normal & Normal & Lento & Lento & $\begin{array}{c}\text { Muy } \\
\text { Lento }\end{array}$ & $\begin{array}{c}\text { Muy } \\
\text { Lento }\end{array}$ \\
\hline & $\begin{array}{c}\text { Lluvia } \\
\text { Débil }\end{array}$ & $\begin{array}{c}\text { Muy } \\
\text { Rápido }\end{array}$ & Rápido & Lento & Normal & Normal & Lento & Lento & $\begin{array}{c}\text { Muy } \\
\text { Lento }\end{array}$ & $\begin{array}{c}\text { Muy } \\
\text { Lento }\end{array}$ \\
\hline & $\begin{array}{c}\text { Lluvia } \\
\text { Normal } \\
\end{array}$ & Rápido & Normal & Lento & Normal & Lento & $\begin{array}{l}\text { Muy } \\
\text { Lento }\end{array}$ & $\begin{array}{c}\text { Muy } \\
\text { Lento }\end{array}$ & $\begin{array}{c}\text { Muy } \\
\text { Lento }\end{array}$ & $\begin{array}{c}\text { Ext. } \\
\text { Lento }\end{array}$ \\
\hline & $\begin{array}{l}\text { Lluvia } \\
\text { Fuerte }\end{array}$ & Normal & Lento & $\begin{array}{c}\text { Muy } \\
\text { Lento }\end{array}$ & Lento & Lento & $\begin{array}{c}\text { Muy } \\
\text { Lento }\end{array}$ & $\begin{array}{c}\text { Muy } \\
\text { Lento }\end{array}$ & $\begin{array}{c}\text { Ext. } \\
\text { Lento }\end{array}$ & $\begin{array}{c}\text { Ext. } \\
\text { Lento }\end{array}$ \\
\hline & $\begin{array}{l}\text { Lluvia } \\
\text { muy } \\
\text { fuerte }\end{array}$ & Lento & Lento & $\begin{array}{c}\text { Muy } \\
\text { Lento }\end{array}$ & $\begin{array}{c}\text { Muy } \\
\text { Lento }\end{array}$ & $\begin{array}{c}\text { Muy } \\
\text { Lento }\end{array}$ & $\begin{array}{c}\text { Ext. } \\
\text { Lento }\end{array}$ & $\begin{array}{c}\text { Ext. } \\
\text { Lento }\end{array}$ & $\begin{array}{c}\text { Ext. } \\
\text { Lento }\end{array}$ & $\begin{array}{c}\text { Ext. } \\
\text { Lento }\end{array}$ \\
\hline & $\begin{array}{c}\text { Lluvia } \\
\text { Intensa }\end{array}$ & Lento & Lento & $\begin{array}{c}\text { Muy } \\
\text { Lento }\end{array}$ & $\begin{array}{c}\text { Muy } \\
\text { Lento }\end{array}$ & $\begin{array}{c}\text { Muy } \\
\text { Lento }\end{array}$ & $\begin{array}{c}\text { Ext. } \\
\text { Lento }\end{array}$ & $\begin{array}{c}\text { Ext. } \\
\text { Lento }\end{array}$ & $\begin{array}{c}\text { Ext. } \\
\text { Lento }\end{array}$ & $\begin{array}{c}\text { Ext. } \\
\text { Lento }\end{array}$ \\
\hline
\end{tabular}

\subsection{Motor de inferencia}

El motor o mecanismo de inferencia es el proceso que, a partir de los valores difusos de entrada y de la base de reglas difusas [14], nos permite calcular los valores difusos de los conjuntos que forman la variable de salida. En la Figura 5 se presenta la variable de salida (Ajuste Difuso).

Otro concepto importante de lógica difusa es la relación difusa. Una relación difusa representa el grado de presencia o ausencia de asociación, interacción o interconexión entre elementos de dos o más conjuntos difusos. La relación difusa es un conjunto difuso en el espacio producto (llamado producto cartesiano). Si las relaciones difusas pertenecen a diferentes espacios producto, su composición difusa se define en forma análoga a la composición clásica. Entonces, usando la operación de composición máximo-mínimo, la composición difusa se define como una relación difusa cuya función de pertenencia está dada por la Ecuación 14.

$$
\mu_{R \circ S}(x, z)=\underset{y \in Y}{\vee}\left(\mu_{R}(x, y) \wedge \quad \mu_{S}(y, z)\right),
$$


donde: $\mu$ representa el grado de pertenencia al conjunto difuso de la variable de salida, $\checkmark$ representa la unión (operador máximo) y $\wedge$ la intersección (operador mínimo).

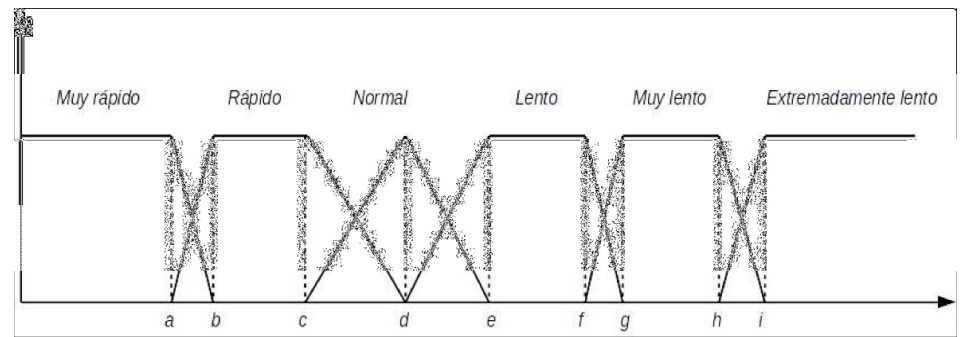

Fig. 5 Conjuntos difusos de la variable de salida: Ajuste Difuso.

\subsection{Desdifusificación}

A partir del proceso de inferencia se obtienen los grados de pertenencia a los conjuntos difusos de la variable de salida, los que necesitan ser convertidos por un proceso de Desdifusificación, es decir, convertir los valores difusos de la variable difusa de salida a valores concretos que puedan ser procesados. En el modelo propuesto representa el ajuste que se multiplicará por el valor de la arista de la red (tiempo de recorrido), obteniendo un valor más aproximado a lo real. Para convertir las salidas difusas en salidas reales se utiliza el método del centro geométrico o centroide, el que consiste en los siguientes cuatro pasos [8]:

Paso 1. Descomponer en figuras regulares la salida difusa, los grados de pertenencia del conjunto difuso forman el límite de las áreas. Para este paso, se analiza la relación que existe entre dos conjuntos difusos adyacente, con función de pertenencia lineal. De este análisis se define dos casos.

a) El primer caso implica que el grado de pertenencia al primer conjunto difuso es menor o igual al valor de pertenencia al segundo conjunto, ver Ecuación 15. En la Figura 6 se observa el área formada y las figuras regulares en las que se divide cuando se cumple el caso 1

$$
\mu_{1}(A D) \leq \mu_{2}(A D)
$$

$\mathrm{AD}=$ Ajuste Difuso.

$\mu_{l}(A D)=$ Función de pertenencia del primer conjunto difuso.

$\mu_{l}(A D)=$ Función de pertenencia del segundo conjunto difuso.

b) En el segundo caso, el primer conjunto difuso es mayor que el segundo conjunto, ver Ecuación 16. En la Figura 7 se observa el área formada y las figuras regulares en las que se divide cuando se cumple el caso 2.

$$
\mu_{1}(A D)>\mu_{2}(A D)
$$

En ambos casos se forman cuatro áreas, los cuales se agrupan para después aplicar los pasos 2, 3 y 4 . 
Eduardo Chandomí-Castellanos, Elías N. Escobar-Gómez, Sabino Velázquez-Trujillo, et al.

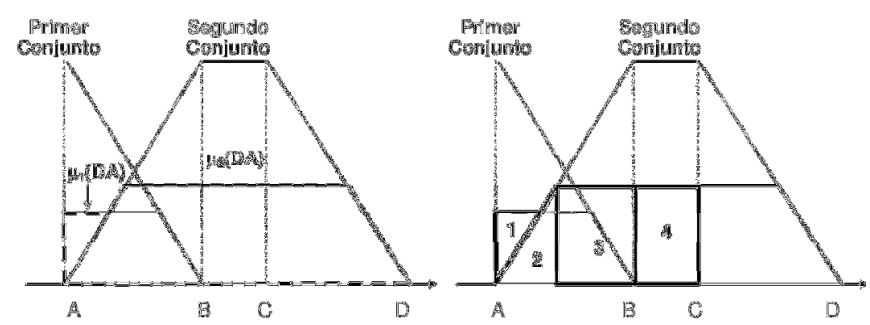

Fig. 6. Área formada y su descomposición en figuras regulares, caso 1.
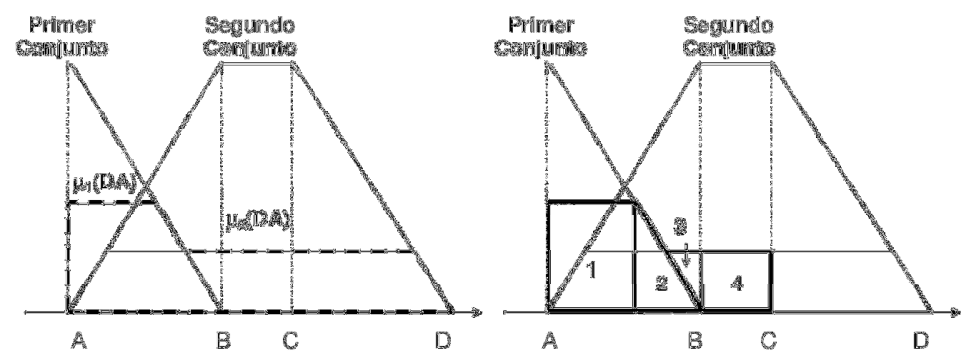

Fig. 7. Área formada y su descomposición en figuras regulares, caso 2.

Paso 2. Calcular la superficie o área de cada figura regular obtenida en el paso 1, aplicando las Ecuaciones 17-20 a los grupos formados.

$$
\begin{gathered}
S\left(A_{i}\right)=\left\{\begin{array}{cc}
\frac{\left[\mu_{1}(A D)\right]^{2}}{2}, \quad \mu_{1}(A D) \leq \mu_{2}(A D), \\
\mu_{1}(A D)\left[1-\mu_{1}(A D)(B-A)\right], \quad \mu_{1}(A D)>\mu_{2}(A D) .
\end{array}\right. \\
S\left(A_{i}\right)= \begin{cases}\frac{\left[\mu_{2}(A D)\right]^{2}(B-A)}{2}, & \mu_{1}(A D) \leq \mu_{2}(A D), \\
\frac{\left[\mu_{1}(A D)\right]^{2}(B-A)}{2}, & \mu_{1}(A D)>\mu_{2}(A D) .\end{cases} \\
S\left(A_{i}\right)=\left\{\begin{array}{cc}
\mu_{2}(A D)\left[1-\mu_{2}(A D)(B-A)\right], & \mu_{1}(A D) \leq \mu_{2}(A D), \\
\frac{\left[\mu_{2}(A D)\right]^{2}(B-A)}{2}, & \mu_{1}(A D)>\mu_{2}(A D) . \\
S\left(A_{i}\right)=\mu_{c}(A D)(C-B) .
\end{array}\right.
\end{gathered}
$$

Paso 3. Determinar el centroide de cada figura, aplicando las Ecuaciones 21-24. 


$$
\begin{gathered}
C\left(A_{i}\right)=\left\{\begin{array}{cc}
A+\frac{(B-A)\left[\mu_{1}(A D)\right]}{3}, & \mu_{1}(A D) \leq \mu_{2}(A D), \\
A+\frac{(B-A)\left[1-\mu_{1}(A D)\right]}{2}, & \mu_{1}(A D)>\mu_{2}(A D) .
\end{array}\right. \\
C\left(A_{i}\right)= \begin{cases}A+\frac{2(B-A)\left[\mu_{2}(A D)\right]}{3}, & \mu_{1}(A D) \leq \mu_{2}(A D), \\
B-\frac{2(B-A)\left[\mu_{1}(A D)\right]}{3}, & \mu_{1}(A D)>\mu_{2}(A D) .\end{cases} \\
C\left(A_{i}\right)=\left\{\begin{array}{cc}
B-\frac{(B-A)\left[1-\mu_{2}(A D)\right]}{2}, & \mu_{1}(A D) \leq \mu_{2}(A D), \\
B-\frac{(B-A)\left[\mu_{2}(A D)\right]}{3}, & \mu_{1}(A D)>\mu_{2}(A D) .
\end{array}\right. \\
C\left(A_{i}\right)=\frac{B+C}{2} .
\end{gathered}
$$

Paso 4. Calcular el centroide total, ver Ecuación 25 como resultado del proceso de desdifusificación, se obtiene un valor real considerado ajuste difuso el cual se multiplica por el valor de la arista, para obtener un valor aproximado a lo real.

$$
C t=\frac{\sum_{i=1}^{n}\left\{\left[S\left(A_{i}\right)\right]\left[C\left(A_{i}\right)\right]\right\}}{\sum_{i=1}^{n} S\left(A_{i}\right)}
$$

\subsection{Selección de la ruta más corta}

Después de considerar la incertidumbre relacionada con los tiempos de recorrido de cada arista de la red se resuelve el problema de la ruta más corta con resultados más precisos; para el cálculo de la ruta más corta se utiliza el algoritmo de Floyd.

\section{Análisis de resultados}

\subsection{Selección de la ruta más corta}

Para el caso de estudio se calcula la ruta más corta de una sección de la ciudad de Tuxtla Gutiérrez, Chiapas, México del primer cuadro de la ciudad que comprende 21 calles y 21 avenidas dando un total de 441 esquinas (nodos) y 884 calles (aristas). Para el tiempo de recorrido se considera las distancias y las velocidades establecidas para cada calle o avenida analizando el estado de las calles, las zonas con tráfico y la precipitación pluvial; los valores para cada variable difusa se presentan en la Tabla 2 (por falta de espacio se muestran los primeros y últimos valores). En el estudio se considera la determinación de la ruta más corta del nodo 1 al nodo 108 .

Para el análisis de los tiempos de recorrido se les aplicó un ajuste de tiempos al modelo propuesto y al modelo con funciones de pertenencia triangular y trapezoidal, para las variables: estado de las calles (Figura 8a), zona de tráfico (Figura 8b) e 
intensidad de lluvia (figura 8c), que suelen ser las funciones de pertenencia usadas comúnmente por su fácil desarrollo y evaluación.

Basado en el método propuesto se desarrolla un programa en MATLAB $®$. Con este programa se calcula el ajuste de los tiempos de recorrido; al programa se le realizan modificaciones en las funciones de pertenencia para determinar el ajuste considerando las funciones mostradas en la Figura 8.

Los resultados de comparación se muestran en la Tabla 3. Los resultados de ambos modelos se comparan con los tiempos obtenidos en el muestreo, con el objetivo de comparar el error porcentual absoluto medio de los modelos con funciones de pertenecía calculadas de manera experimental y el error porcentual absoluto medio de los modelos con funciones clásica, ver Tabla 4.

Tabla 2. Unidad de medida de las variables difusas.

\begin{tabular}{|c|c|c|c|c|c|}
\hline \multicolumn{2}{|c|}{ Estado de la calle } & \multicolumn{2}{|c|}{ Zona de trafico } & \multicolumn{2}{|c|}{ Intensidad de Lluvia } \\
\hline $\begin{array}{l}\text { Unidad de } \\
\text { medida }\end{array}$ & Descripción & $\begin{array}{l}\text { Unidad de } \\
\text { medida }\end{array}$ & Descripción & $\begin{array}{l}\text { Unidad de } \\
\text { medida }\end{array}$ & Descripción \\
\hline 1 & Un tope pequeño & 1 & $\begin{array}{l}\text { Banco } 5 \text { de la } \\
\text { mañana }\end{array}$ & $0 \mathrm{ml}$ & Sin lluvia \\
\hline 2 & Un bache pequeño & 2 & $\begin{array}{l}\text { Banco } 6 \text { de la } \\
\text { mañana }\end{array}$ & $1 \mathrm{ml}$ & \multirow{7}{*}{$\begin{array}{l}\text { Cantidad de } \\
\text { lluvia en } \\
\text { mililititros }\end{array}$} \\
\hline 3 & Dos topes pequeños & 3 & $\begin{array}{l}\text { Banco } 7 \text { de la } \\
\text { mañana }\end{array}$ & \multirow{5}{*}{$\vdots$} & \\
\hline 4 & Dos baches pequeños & 4 & $\begin{array}{l}\text { Banco } 6 \text { de la } \\
\text { tarde }\end{array}$ & & \\
\hline 5 & $\begin{array}{c}\text { Alcantarillado } \\
\text { parcialmente en mal } \\
\text { estado }\end{array}$ & 5 & $\begin{array}{l}\text { Banco } 7 \text { de la } \\
\text { tarde }\end{array}$ & & \\
\hline$:$ & 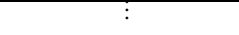 & $:$ & : & & \\
\hline 26 & Tres baches grandes & 71 & $\begin{array}{l}2 \text { de la tarde } \\
\text { cruceros }\end{array}$ & & \\
\hline 27 & $\begin{array}{l}\text { Deformación total de la } \\
\text { calle }\end{array}$ & 72 & $\begin{array}{l}2 \text { de la tarde } \\
\text { escuelas }\end{array}$ & $60 \mathrm{ml}$ más & \\
\hline
\end{tabular}

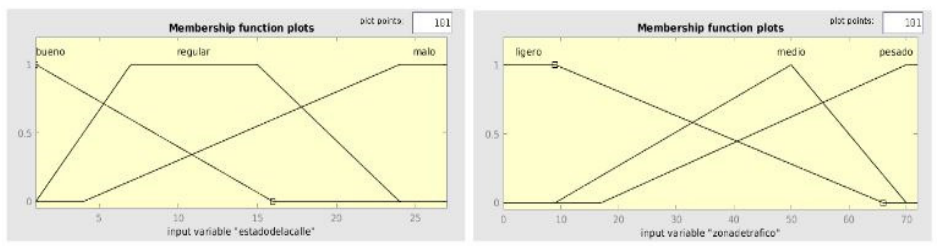

(a) Estado de la calle

(b) Zona de Tráfico

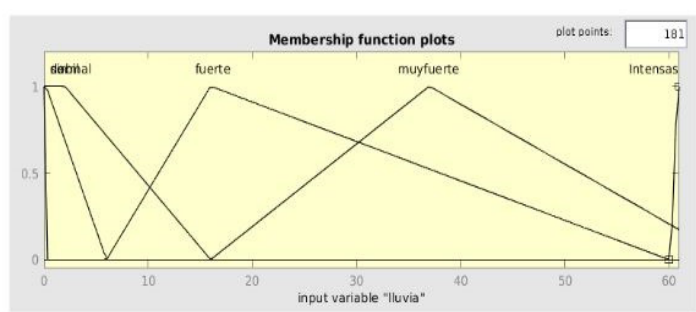

(c) Lluvia

Fig. 8. Funciones de pertenencia trapezoidal y triangular. 
Modelo para la determinación de la ruta más corta con funciones experimentales para arcos difusos

Tabla 3. Comparación de funciones experimentales y clásicas.

\begin{tabular}{|c|c|c|c|c|c|c|c|c|c|c|}
\hline & \multicolumn{2}{|c|}{ Variables difusas } & \multicolumn{3}{|c|}{ Experimental } & \multicolumn{3}{c|}{ Clásicas } & $\begin{array}{c}\text { Valor } \\
\text { Real } \\
\text { (min) }\end{array}$ \\
\hline & IL & EC & ZT & $\begin{array}{c}\text { Ajuste } \\
\text { Difuso }\end{array}$ & $\begin{array}{c}\text { Salida } \\
(\mathrm{min})\end{array}$ & Diferencia & $\begin{array}{c}\text { Ajuste } \\
\text { Difuso }\end{array}$ & $\begin{array}{c}\text { Salida } \\
(\mathrm{min})\end{array}$ & Diferencia & 3.3 \\
\hline 1 & 0 & 1 & 1 & 0.58 & 2.9 & 0.4 & 0.61 & 3.05 & 0.25 & 2.6 \\
\hline 2 & 0 & 7 & 1 & 0.88 & 2.552 & 0.048 & 1.47 & 4.263 & 1.663 & 0.25 \\
\hline 3 & 0 & 1 & 1 & 0.75 & 0.2625 & 0.0125 & 0.78 & 0.273 & 0.023 & 0.25 \\
\hline 4 & 0 & 1 & 21 & 0.75 & 0.57 & 0.01 & 0.78 & 0.5928 & 0.0128 & 0.58 \\
\hline 5 & 0 & 1 & 21 & 0.75 & 1.695 & 0.025 & 0.78 & 1.7628 & 0.0928 & 1.67 \\
\hline 6 & 0 & 1 & 1 & 0.58 & 1.1078 & 0.2622 & 0.61 & 1.1651 & 0.2049 & 1.37 \\
\hline 7 & 0 & 1 & 1 & 0.58 & 0.87 & 0.02 & 0.61 & 0.915 & 0.065 & 0.85 \\
\hline & & & & Total: & 9.95 & 1.4477 & Total: & 12.02 & 3.7115 & 10.62 \\
\hline
\end{tabular}

Tabla 4. Datos para comparar el error porcentual absoluto medio.

\begin{tabular}{|c|c|c|c|c|}
\hline $\begin{array}{c}\text { Tiempo de } \\
\text { recorrido } \\
\text { muestreado }\end{array}$ & $\begin{array}{c}\text { Tiempo con } \\
\text { funciones } \\
\text { experimentales } \\
\text { (FE) }\end{array}$ & $\begin{array}{c}\text { Porcentaje de } \\
\text { error absoluto } \\
\text { con FE }\end{array}$ & $\begin{array}{c}\text { Tiempo con } \\
\text { funciones } \\
\text { clásicas (FC) }\end{array}$ & $\begin{array}{c}\text { Porcentaje de } \\
\text { error absoluto } \\
\text { con FC }\end{array}$ \\
\hline 3.3 & 2.9 & $12 \%$ & 3.05 & $8 \%$ \\
\hline 2.6 & 2.552 & $2 \%$ & 4.263 & $64 \%$ \\
\hline 0.25 & 0.2625 & $5 \%$ & 0.273 & $9 \%$ \\
\hline 0.58 & 0.57 & $2 \%$ & 0.5928 & $2 \%$ \\
\hline 1.67 & 1.695 & $1 \%$ & 1.7628 & $6 \%$ \\
\hline 1.37 & 1.1078 & $19 \%$ & 1.1651 & $15 \%$ \\
\hline 0.85 & 0.87 & $2 \%$ & 0.915 & $8 \%$ \\
\hline \multicolumn{2}{r|}{ Media } & $6 \%$ & & $16 \%$ \\
\hline Varianza & $0.47 \%$ & & $4.65 \%$ \\
\hline
\end{tabular}

Se observa en la Tabla 4 que el error porcentual absoluto medio es menor en el modelo con funciones de pertenencia experimentales, también es menor la varianza; por lo que concluimos que el modelo con funciones de pertenencia experimentales predice mejor los tiempos de recorrido que el modelo con funciones de pertenencia clásicas.

\section{Conclusión}

El problema de la ruta más corta es muy importante para aplicaciones prácticas. Sin embargo, el uso de lógica difusa para considerar los tiempos de recorrido como un valor no determinista mejora los resultados obtenidos.

En este artículo, la implementación de la lógica difusa permite modelar la incertidumbre del problema de la optimización de rutas al entregar productos en la red de carreteras urbanas a través de tres variables lingüísticas: Estado de las Calles, Zonas de Tráfico e Intensidad de Lluvia. Con el cálculo de las funciones de pertenencia se pretende imitar la experiencia de un experto en distribución, tomando en cuenta factores que disminuyen el tiempo de entrega.

Para determinar la ruta más corta dada una serie de nodos o esquina, primero se evalúa la incertidumbre de cada arco de la red, aplicando un sistema de inferencia difuso con funciones de pertenencia calculadas de manera experimental, posteriormente con los algoritmos de ruta más corta se obtiene el recorrido con el menor tiempo. Se 
concluye que las funciones de pertenencia calculadas de maneras experimentales y basadas en experiencia del experto, se apegan más a los datos reales en comparación con las funciones de pertenencia clásicas.

\section{Referencias}

1. Arreola Risa, J.S., Arreola Risa, A.: Programación lineal: Una introducción a la toma de decisiones cuantitativa. Internacional Thomson Editores (2003)

2. Ballou, R.H.: Logística: Administración de la cadena de suministro. Pearson Educación (2004)

3. Bellman, R.: On a routing problem. Tech. rep., DTIC Document (1956)

4. Bienstock, C.C., Mentzer, J.T., Bird, M.M.: Measuring physical distribution service quality. Journal of the Academy of Marketing Science 25(1), 31-44 (1996)

5. Cormen, T.H., Leiserson, C.E., Rivest, R.L., Stein, C.: Introduction to algorithms, vol. 6. MIT press Cambridge (2001)

6. Deng, Y., Chen, Y., Zhang, Y., Mahadevan, S.: Fuzzy dijkstra algorithm for shortest path problem under uncertain environment. Applied Soft Computing 12(3), 1231-1237 (2012)

7. Dijkstra, E.W.: A note on two problems in connexion with graphs. Numerische mathematik 1(1), 269-271 (1959)

8. Escobar-Gómez, E., Díaz-Núñez, J., Taracena-Sanz, L.: Modelo para el ajuste de pronósticos agregados utilizando lógica difusa. Ingeniería, investigación y tecnología 11(3), 289$302(2010)$

9. Floyd, R.W.: Algorithm 97: shortest path. Communications of the ACM 5(6), 345 (1962)

10. Gutiérrez, T.R., Read, J.S.B., De los Ríos, J.A.S., Gándara, L.C.L., Hernández, J.C.P.: Desarrollo de un controlador difuso y su interfaz de usuario para regulación de potencia en un reactor de investigación (2007)

11. Ji, X.: Models and algorithm for stochastic shortest path problem. Applied Mathematics and Computation 170(1), 503-514 (2005)

12. Kotler, P., Armstrong, G.: Fundamentos de marketing. Pearson Educación (2003)

13. López, M., Viñas Rubio, J.M.: Nuevo manual de uso de términos meteorológicos de aemet (2015)

14. Mamdani, E.H., Assilian, S.: An experiment in linguistic synthesis with a fuzzy logic controller. International journal of man-machine studies 7(1), 1-13 (1975)

15. Martín del Brío, B., Sanz Molina, A.: Redes neuronales y sistemas difusos. Alfaomega Ra$\mathrm{Ma}(2002)$

16. Orozco-Soto, S.M., Fernández, J.C.R.: Control par calculado difuso basado en pasividad para seguimiento de trayectorias de robots manipuladores. Research in Computing Science 91, pp. 131-141 (2015)

17. Ramos, N.T., Hernández, J.A.R., Cerda, D.A.S., Sánchez, A., López, A.Q.R.: Diagnóstico de fallas en centrales termoeléctricas utilizando modelado neuronal y lógica difusa (2007)

18. Ross, T.J.: Fuzzy logic with engineering applications. John Wiley \& Sons (2009)

19. Shukla, K.T.: Fuzzy Floyd's algorithm to find shortest route between nodes under uncertain environment. International Journal of Mathematics and Computer Applications Research (IJMCAR) 1(3), 43-54 (2013)

20. Zadeh, L.A.: Fuzzy sets as a basis for a theory of possibility. Fuzzy sets and systems 1(1), 328 (1978) 